\title{
Area-law versus Rényi and Tsallis black hole entropies
}

\author{
Shin'ichi Nojiri, ${ }^{1,2}$ Sergei D. Odintsov, ${ }^{3,4,5,6}$ and Valerio Faraoni $\odot^{7}$ \\ ${ }^{1}$ Department of Physics, Nagoya University, Nagoya 464-8602, Japan \\ ${ }^{2}$ Kobayashi-Maskawa Institute for the Origin of Particles and the Universe, Nagoya University, \\ Nagoya 464-8602, Japan \\ ${ }^{3}$ Institució Catalana de Recerca i Estudis Avançats (ICREA), \\ Passeig Lluís Companys, 23, 08010 Barcelona, Spain \\ ${ }^{4}$ Institute of Space Sciences (IEEC-CSIC), C.Can Magrans s/n, 08093 Barcelona, Spain \\ ${ }^{5}$ Department of Physics, Kazan Federal University, Kazan 420008, Russia \\ ${ }^{6}$ International Laboratory of Theoretical Cosmology, Tomsk State University of Control Systems and \\ Radioelectronics(TUSUR), 634050 Tomsk, Russia \\ ${ }^{7}$ Department of Physics \& Astronomy, Bishop's University, 2600 College Street, \\ Sherbrooke, Québec J1M 1Z7, Canada
}

(Received 10 September 2021; accepted 20 September 2021; published 6 October 2021)

\begin{abstract}
The Rényi and Tsallis entropies are discussed as possible alternatives to the Bekenstein-Hawking arealaw entropy. It is pointed out how replacing the entropy notion, but not the Hawking temperature and the thermodynamical energy may render the whole black hole thermodynamics inconsistent. The possibility to relate the Rényi and Tsallis entropies with the quantum gravity corrected Bekenstein-Hawking entropy is discussed.
\end{abstract}

DOI: 10.1103/PhysRevD.104.084030

\section{INTRODUCTION}

Black hole thermodynamics is one of the most interesting recent discoveries of theoretical physics. Bekenstein [1] argued that the area $A$ of a black hole horizon has the properties of the entropy $\mathcal{S}$ in ordinary thermodynamics and must be proportional to it (the proportionality factor was determined later). However, the similarity between black hole area and thermodynamical entropy did not make sense initially because it was believed that black holes are cold objects, until Hawking discovered that the Schwarzschild black hole emits quantum radiation with a blackbody spectrum at temperature $T_{\mathrm{H}}=\frac{1}{8 \pi G M}$ (now called the Hawking temperature), where $M$ is the Schwarzschild mass of the black hole [2]. Here we use geometrized units in which the speed of light $c$, the Boltzmann constant $K_{B}$, and the reduced Planck constant $\hbar$ are unity.

Hawking's discovery of black hole radiation originates from the application of quantum field theory to curved spacetime and, by extension, it implies that all black holes must radiate, making them thermal objects and completing Bekenstein's suggestion. The Bekenstein-Hawking entropy $\mathcal{S}=A / 4$ and the Hawking temperature $T_{\mathrm{H}}$ allow for the construction of a self-consistent black hole thermodynamics ([3], see [4-6] for reviews), which is now an important part of modern theoretical physics. Adding a cosmological constant, as in the Schwarzschild-de Sitter/Kottler and Schwarzschild-anti-de Sitter black holes, adds richness to the thermodynamical behavior of black holes. In addition to creating multiple horizons which could be viewed as thermodynamical subsystems, it leads to the possibility of the Hawking-Page phase transition [7], which was later interpreted in the context of the AdS/CFT correspondence as the counterpart of the deconfinement transitions for the conformal field theory living on the anti-de Sitter boundary $[8,9]$. The negative cosmological constant turns out to play the role of the pressure, to be added to the thermodynamical picture [10], thus extending the structure of the phase space and making phase transitions possible. In this context, a rich literature on "black hole chemistry" has emerged and black hole thermodynamics has taken a new lease on life (e.g., [11-19] and references therein).

Originally, it came as a surprise that the BekensteinHawking entropy is proportional to the black hole area and not to its volume, as in ordinary thermodynamics where entropy is an extensive quantity proportional to the mass, and then to the volume, of a system. This feature largely remains a mystery [20]. Recent literature has discussed the possibility of replacing the Bekenstein-Hawking entropy with other entropy notions based on nonextensive statistics [20-37], such as the Rényi [38] and Tsallis [39] entropies. However, changing the entropy notion is risky because entropy enters many thermodynamical equations and other quantities need to be modified in order to keep the whole construction of thermodynamics self-consistent. While modifying entropy is challenging and many authors have focused on this task, here we point out the risks inherent in these modifications for related thermodynamics. 
In particular, it is problematic to modify the Hawking temperature of blackbody radiation based only on nonextensive statistics, while there are independent arguments pointing toward the "correct" choice of thermodynamical energy and black hole mass. When all these aspects are considered together, it turns out to be quite difficult to replace the Bekenstein-Hawking entropy based solely on the idea of nonextensive statistics while keeping black hole thermodynamics self-consistent.

In this work we follow the notation of Ref. [5]: the metric signature is -+++ and the units are such that the speed of light $c$, the Boltzmann constant $K_{B}$, and the reduced Planck constant $\hbar$ are unity.

\section{STANDARD BLACK HOLE THERMODYNAMICS}

The geometry of the Schwarzschild black hole is described by the line element [5]

$d s^{2}=-f(r) d t^{2}+\frac{d r^{2}}{f(r)}+r^{2} d \Omega_{(2)}^{2}, \quad f(r) \equiv 1-\frac{2 G M}{r}$,

where $G$ is Newton's constant, $M$ is the black hole mass, and $d \Omega_{(2)}^{2}=d \vartheta^{2}+\sin ^{2} \vartheta d \varphi^{2}$ is the line element on the unit two-sphere. The black hole event horizon is located at the Schwarzschild radius

$$
r_{\mathrm{H}}=2 G M \text {. }
$$

By considering quantum field theory on the spacetime with this horizon, Hawking discovered that the Schwarzschild black hole radiates with a blackbody spectrum at the temperature [2]

$$
T_{\mathrm{H}}=\frac{1}{8 \pi G M} .
$$

The Hawking temperature can be also understood geometrically. When $r \sim r_{\mathrm{H}}$, we define $\delta r$ by $r \equiv r_{\mathrm{H}}+\delta r$. Then by Wick-rotating the time coordinate $t \rightarrow i \tau$, the line element (2.1) is recast as

$$
d s^{2} \simeq \frac{\delta r}{r_{\mathrm{H}}} d \tau^{2}+\frac{r_{\mathrm{H}}}{\delta r} d(\delta r)^{2}+r_{\mathrm{H}}^{2} d \Omega_{(2)}^{2} .
$$

We further define a new radial coordinate $\rho$ by $d \rho=d(\delta r) \sqrt{r_{\mathrm{H}} / \delta r}$, that is

$$
\rho=2 \sqrt{r_{\mathrm{H}} \delta r} \quad \text { or } \quad \delta r=\frac{\rho^{2}}{4 r_{\mathrm{H}}},
$$

in terms of which the line element (2.4) becomes

$$
d s^{2} \simeq \frac{\rho^{2}}{4 r_{\mathrm{H}}^{2}} \rho^{2} d \tau^{2}+d \rho^{2}+r_{\mathrm{H}}^{2} d \Omega_{(2)}^{2} .
$$

In order to avoid the conical singularity in the Wick-rotated Euclidean space around $\rho \sim 0$, we require the periodicity of the Euclidean time coordinate $\tau$

$$
\frac{\tau}{2 r_{\mathrm{H}}} \sim \frac{\tau}{2 r_{\mathrm{H}}}+2 \pi
$$

Because the inverse of the period $t_{0}$ of the Euclidean time coordinate corresponds to the temperature, as in the Euclidean path integral formulation of the finite temperature field theory for any field $\phi$,

$$
\int[D \phi] \mathrm{e}^{\int_{0}^{t_{0}} d t L(\phi)}=\operatorname{Tr}\left(\mathrm{e}^{-t_{0} H}\right)=\operatorname{Tr}\left(\mathrm{e}^{-\frac{H}{T}}\right),
$$

one finds that the Schwarzschild black hole has temperature $T$, which is nothing but the Hawking temperature (2.3),

$$
T=\frac{1}{4 \pi r_{\mathrm{H}}}=\frac{1}{8 \pi G M} \equiv T_{\mathrm{H}} .
$$

Thus, the Hawking temperature can be obtained solely from the geometry of the spacetime endowed with the event horizon.

We can derive also the entropy $\mathcal{S}$ from the geometrical point of view. Eq. (2.8) tells us that the partition function $Z(T)$ and the free energy $F(T)$ are given by

$$
\mathrm{e}^{-\frac{F(T)}{T}}=Z(T)=\operatorname{Tr}\left(\mathrm{e}^{-\frac{H}{T}}\right)=\int[D \phi] \mathrm{e}^{S(\phi)},
$$

with the periodic boundary condition that the Euclidean time has period $1 / T$. In Eq. (2.10), $S(\phi)=\int_{0}^{t_{0}} d t L(\phi)$ is the Euclidean action. In the low-temperature regime when $T$ is sufficiently small, the path integral (2.10) can be estimated as

$$
\int[D \phi] \mathrm{e}^{S(\phi)} \sim \mathrm{e}^{S\left(\phi_{\mathrm{cl}}\right)}
$$

in the WKB approximation, where $\phi_{\mathrm{cl}}$ is a classical solution of the field equations given by the Euclidean action $S(\phi)$. Then Eq. (2.10) allows us to estimate the free energy $F$ which, in turn, gives the entropy $\mathcal{S}$ by using the thermodynamical relations.

In order to estimate the free energy $F$, we consider the Schwarzschild-anti-de Sitter geometry

$$
\begin{gathered}
d s^{2}=-f(r) d t^{2}+\frac{d r^{2}}{f(r)}+r^{2} d \Omega_{(2)}^{2}, \\
f(r) \equiv 1-\frac{2 G M}{r}+\frac{r^{2}}{l^{2}}
\end{gathered}
$$


where $\Lambda=-3 / l^{2}$ is the cosmological constant (the reason why we consider the Schwarzschild-anti-de Sitter spacetime instead of the Schwarzschild one with the flat Minkowski background will be explained later).

We now rewrite the function $f(r)$ in the form

$$
\begin{array}{r}
f(r)=\frac{\left(r-r_{\mathrm{H}}\right)\left(r+\frac{r_{\mathrm{H}}}{2}+i a\right)\left(r+\frac{r_{\mathrm{H}}}{2}-i a\right)}{l^{2} r}, \\
r_{\mathrm{H}}\left(\frac{r_{\mathrm{H}}^{2}}{4}+a^{2}\right)=2 G M l^{2}, \quad-\frac{3 r_{\mathrm{H}}^{2}}{4}+a^{2}=l^{2} .
\end{array}
$$

Eliminating $a$ in the last two equations yields

$$
r_{\mathrm{H}}\left(r_{\mathrm{H}}^{2}+l^{2}\right)=2 G M l^{2} \text {. }
$$

When $r \sim r_{\mathrm{H}}, f(r)$ behaves as

$$
f(r) \sim \frac{\frac{9 r_{\mathrm{H}}^{2}}{4}+a^{2}}{l^{2} r_{\mathrm{H}}}\left(r-r_{\mathrm{H}}\right)
$$

and the Hawking temperature is

$T_{\mathrm{H}} \simeq \frac{\frac{9 r_{\mathrm{H}}^{2}}{4}+a^{2}}{4 \pi l^{2} r_{\mathrm{H}}}=\frac{3 r_{\mathrm{H}}^{2}+l^{2}}{4 \pi l^{2} r_{\mathrm{H}}}=\frac{1}{4 \pi l^{2}}\left(3 r_{\mathrm{H}}+\frac{l^{2}}{r_{\mathrm{H}}}\right)$,

where we have used the last equation (2.15) to substitute for $a$.

After the Wick rotation, the action becomes

$$
\begin{aligned}
S & =\frac{1}{16 \pi G} \int d^{4} x \sqrt{-g}\left(R+\frac{6}{l^{2}}\right) \\
& =-\frac{3}{2 G l^{2}} \int_{0}^{1 / T_{\mathrm{H}}} d t \int_{r_{\mathrm{H}}}^{L} d r r^{2} \\
& =\frac{r_{\mathrm{H}}^{3}-L^{3}}{2 G l^{2} T_{\mathrm{H}}},
\end{aligned}
$$

where we have introduced a cutoff $L$ to regulate the divergence of the action (2.19). It is instructive to consider the difference between the action of the Schwarzschildanti-de Sitter spacetime and that of the pure anti-de Sitter spacetime. We determine the period of the Euclidean time $1 / \tilde{T}_{\mathrm{H}}$ in anti-de Sitter space so that the physical length (between $t=0$ and $t=1 / \tilde{T}_{\mathrm{H}}$ ) equals the physical length (between $t=0$ and $t=1 / T_{\mathrm{H}}$ ) in the Schwarzschildanti-de Sitter spacetime:

$$
\left(1-\frac{2 G M}{L}+\frac{L^{2}}{l^{2}}\right)^{1 / 2} \frac{1}{T_{\mathrm{H}}}=\left(1+\frac{L^{2}}{l^{2}}\right)^{1 / 2} \frac{1}{\tilde{T}_{\mathrm{H}}},
$$

or

$$
\frac{1}{\tilde{T}_{\mathrm{H}}}=\frac{1}{T_{\mathrm{H}}} \sqrt{1-\frac{2 G M}{L\left(1+\frac{L^{2}}{l^{2}}\right)}} \sim \frac{1}{T_{\mathrm{H}}}\left(1-\frac{G M l^{2}}{L^{3}}\right) .
$$

Then, the action $S_{\text {AdS }}$ for anti-de Sitter spacetime is

$$
\begin{aligned}
S_{\mathrm{AdS}} & =-\frac{3}{2 G l^{2}} \int_{0}^{1 / \tilde{T}_{\mathrm{H}}} d t \int_{0}^{L} d r r^{2}=-\frac{L^{3}}{2 G l^{2} \tilde{T}_{\mathrm{H}}} \\
& \sim-\frac{L^{3}}{2 G l^{2} T_{\mathrm{H}}}+\frac{G M l^{2}}{2 G l^{2} T_{\mathrm{H}}} \\
& =-\frac{L^{3}}{2 G l^{2} T_{\mathrm{H}}}+\frac{r_{\mathrm{h}}\left(r_{\mathrm{H}}^{2}+l^{2}\right)}{4 G l^{2} T_{\mathrm{H}}},
\end{aligned}
$$

where we have used Eq. (2.16). In the limit $L \rightarrow \infty$, the action $S_{\mathrm{BH}}$ for the black hole in anti-de Sitter space reduces to

$$
S_{\mathrm{BH}}=S-S_{\mathrm{AdS}}=\frac{r_{\mathrm{H}}\left(r_{\mathrm{H}}^{2}-l^{2}\right)}{4 G l^{2} T_{\mathrm{H}}}
$$

and the free energy $F$ is

$$
F=-T_{\mathrm{H}} S_{\mathrm{BH}}=-\frac{r_{\mathrm{H}}\left(r_{\mathrm{H}}^{2}-l^{2}\right)}{4 G l^{2}} .
$$

Upon use of the thermodynamical relations

$$
E=F-T_{\mathrm{H}} \frac{d F}{d T_{\mathrm{H}}}, \quad \mathcal{S}=\frac{E-F}{T_{\mathrm{H}}}=-\frac{d F}{d T_{\mathrm{H}}}
$$

for the thermodynamical energy $E$ and the entropy $\mathcal{S}$, we find the entropy

$$
\mathcal{S}=-\frac{d F / d r_{\mathrm{H}}}{d T_{\mathrm{H}} / d r_{\mathrm{H}}}=\frac{\frac{3 r_{\mathrm{H}}^{2}-l^{2}}{4 G l^{2}}}{\frac{1}{4 \pi l^{2}}\left(3-\frac{l^{2}}{r_{\mathrm{H}}^{2}}\right)}=\frac{\pi r_{\mathrm{H}}^{2}}{G}=\frac{A}{4 G},
$$

where $A=4 \pi r_{\mathrm{H}}^{2}$ is the horizon area. The expression (2.26) is valid in the limit of a flat Minkowski background $l^{2} \rightarrow \infty$, thus we obtain the Bekenstein-Hawking entropy from a geometrical viewpoint. We should note, however, that the action (2.19) vanishes in the Minkowski background $l^{2} \rightarrow \infty$, hence, the "Schwarzschild" black hole in anti-de Sitter space with finite $l$ somehow plays the role of the regularization of the Schwarzschild black hole in flat Minkowski background.

Another consideration is in order: restoring the constants, the Hawking temperature and the BekensteinHawking entropy read

$$
T_{\mathrm{H}}=\frac{\hbar c^{3}}{8 \pi G K_{B} M}, \quad \mathcal{S}=\frac{c^{2} A}{4 G \hbar},
$$

then the free energy $F=E-T S$ does not contain the reduced Planck constant $\hbar$ and, in this sense, it is a classical 
quantity like $E$. Nonextensive black hole entropies that are inspired by modified uncertainly principles or quantum gravity corrections should not affect $F$ unless they correct $T_{\mathrm{H}}$. Then, according to Eq. (2.26), the entropy $\mathcal{S}$ is only affected through corrections to the black hole temperature. However, if this temperature remains the Hawking temperature $T_{\mathrm{H}}, \mathcal{S}$ cannot receive corrections, or else also Eq. (2.26) must be modified accordingly. Although a bit hand-waving, ${ }^{1}$ this argument makes the point that thermodynamics (including black hole thermodynamics) must be a consistent theory and modifying this or that thermodynamic quantity ad hoc usually has unwanted consequences for the rest of the theory.

\section{IMPOSSIBILITY OF NONAREA LAW ENTROPY}

In the previous section, we have summarized the derivation of both the Hawking temperature and the Bekenstein-Hawking entropy from the viewpoint of the geometry. As is well known, the area law for the Bekenstein-Hawking entropy [1] can always be obtained if we identify the thermodynamical energy $E$ with the black hole mass $M, E=M$, and the temperature of the system with the Hawking temperature (2.3) [2], $T=T_{\mathrm{H}}$. In fact, the thermodynamical relation $d E=T d \mathcal{S}$ yields

$$
d \mathcal{S}=\frac{d E}{T}=8 \pi G M d M=d\left(4 \pi G M^{2}\right),
$$

that is,

$$
\mathcal{S}=4 \pi G M^{2}+\mathcal{S}_{0},
$$

where $S_{0}$ is an integration constant. If we assume $\mathcal{S}=0$ when $M=0$, that is, in the absence of the black hole, then $\mathcal{S}_{0}=0$ and

$$
\mathcal{S}=\frac{\pi r_{\mathrm{H}}^{2}}{G}=\frac{A}{4 G}
$$

where $A \equiv 4 \pi r_{\mathrm{H}}^{2}$ is the horizon area. Thus, we have shown that the Bekenstein-Hawking entropy (i.e., the area law for the black hole entropy) can be obtained by assuming $E=M$ and $T=T_{\mathrm{H}}$ by using the thermodynamical relation $d \mathcal{S}=d E / T$.

Two questions arise naturally:

(1) Can we identify the thermodynamical energy $E$ with the black hole mass $M$ (i.e., $E=M)$ ? Furthermore, if the black hole is not Schwarzschild nor isolated, there is no Arnowitt-Deser-Misner mass: should then $M$ be the quasilocal mass contained in the horizon sphere, or the "black hole part" of it? If so,

\footnotetext{
${ }^{1}$ In the sense that quantum gravity is expected to correct the Hawking temperature at some level.
}

which quasilocal mass? Several quasilocal mass prescriptions exist in the literature (see Ref. [40] for a review).

(2) Is the temperature of the black hole given by the Hawking temperature, $T=T_{\mathrm{H}}$ ?

To answer the first question, consider the following gedankenexperiment: assume that there is an infalling spherically symmetric shell of dust with mass $M$ and initial radius sufficiently large. By virtue of the Birkhoff theorem [5], the spacetime outside the shell is the Schwarzschild one (2.1), where $M$ in (2.1) is the mass of the shell. Inside the shell, spacetime is empty and flat. The shell collapses, its radius becoming smaller and smaller. When the shell crosses its Schwarzschild radius (2.2), a black hole is formed. The geometry is always asymptotically flat and the shell mass $M$ appearing in the Schwarzschild line element is surely the energy $E$ of the system, $E=M$. This energy is conserved during the collapse of the shell because, due to the Birkhoff theorem, the geometry outside of it does not change during the collapse: the energy of the final black hole must be the mass of the shell. ${ }^{2}$ Therefore, the thermodynamical energy $E$ is the black hole mass $E=M$.

Regarding the mass concept used, the answer is easy for the Schwarzschild black hole: the Schwarzschild mass appearing in the line element is the obvious choice. It coincides with the Misner-Sharp-Hernandez quasilocal mass $M_{\mathrm{MSH}}$ defined in any spherically symmetric spacetime by $[41,42]$

$$
1-\frac{2 G M_{\mathrm{MSH}}}{R}=\nabla^{c} R \nabla_{c} R
$$

where $R$ is the areal radius [which coincides with $r$ in the Schwarzschild case (2.1)]. Other quasilocal masses do not reproduce the standard Hawking temperature and the Bekenstein-Hawking entropy. For example, consider the Brown-York quasilocal energy [43] as a possible candidate to the role of thermodynamical energy. For a spherically symmetric metric of the form

$$
d s^{2}=-N^{2}(t, r) d t^{2}+\frac{d r^{2}}{f(t, r)}+r^{2} d \Omega_{(2)}^{2},
$$

the Brown-York energy is $[40,43]$

$$
E_{\mathrm{BY}}=\frac{r}{G}[1-f(t, r)]
$$

For the Schwarzschild metric, the Brown-York energy is radius-dependent:

\footnotetext{
${ }^{2}$ Due to spherical symmetry, gravitational waves (which are quadrupole to lowest order) are not emitted during the collapse and cannot carry away energy.
} 


$$
E_{\mathrm{BY}}=\frac{r}{G}\left(1-\sqrt{1-\frac{2 M}{r}}\right)
$$

at the Schwarzschild horizon $r=r_{\mathrm{H}}$, we have $E_{\mathrm{BY}}=2 M$. If we identify the thermodynamical energy with the BrownYork energy and the black hole temperature with the Hawking temperature, the relation $T d \mathcal{S}=d E$ yields

$$
d \mathcal{S}_{\mathrm{BY}}=\frac{d E_{\mathrm{BY}}}{T_{\mathrm{H}}}=16 \pi G M d M
$$

and, integrating,

$$
\mathcal{S}_{\mathrm{BY}}=8 \pi M^{2}=\frac{A}{2 G},
$$

which is unphysical. The only way to reconcile the BrownYork energy prescription [43] with the relation $T d \mathcal{S}=d E$ is by introducing the Brown-York temperature $T_{\mathrm{BY}}=2 T_{\mathrm{H}}$, which disagrees with Hawking's fundamental result [2]. One does not see how (or why) Hawking's derivation of the black hole temperature should be modified to make it agree with the Brown-York mass prescription in thermodynamics.

Let us consider now the Schwarzschild-anti-de Sitter black hole (parallel considerations apply to the Schwarzschild-de Sitter case). For the Schwarzschildanti-de Sitter black hole, the Misner-Sharp-Hernandez mass is ${ }^{3}$

$$
M_{\mathrm{MSH}}=M-\frac{r^{2}}{2 G l^{2}},
$$

where it is easy to isolate the black hole contribution from the one due to the negative cosmological constant, and the Hawking temperature and the Bekenstein-Hawking entropy should refer to these contributions (at least in the limit of small black holes). This happens also for more general black holes embedded in a cosmological "background" described by the time-dependent McVittie metric [44] (this geometry contains the Schwarschild-de Sitter/Kottler metric as a special case) $[45,46]$. However, this is not the case for other cosmological black holes, for example for the Sultana-Dyer black hole [47] for which the Misner-Sharp-Hernandez/ Hawking-Hayward mass includes a third term coupling the black hole and the cosmological energies [46]. However it can be argued that, at least for the Sultana solution, the time-dependence of both black hole and cosmological horizons precludes a discussion with equilibrium thermodynamics while, in general, an adiabatic expansion for

\footnotetext{
${ }^{3}$ For the Schwarzschild-anti-de Sitter black hole, the BrownYork energy gives again an unphysical result, i.e., $E_{\mathrm{BY}}=$ $2 M-\frac{r^{3}}{G l^{2}}$.
}

dynamical black holes should recover the Schwarzschild thermodynamics.

It is interesting that the thermodynamics of dynamical apparent horizons (as opposed to static null event horizons) employs the Hawking-Hayward quasilocal energy as the thermodynamical energy of a black hole, which reduces to the Misner-Sharp-Hernandez mass in spherical symmetry [48]. Due to the time-dependence, the first law of thermodynamics must be generalized to include an energy supply vector $[49,50]$. In spherical symmetry, the Clausius definition of entropy coincides with the entropy obtained from Wald's Noether charge method [51,52] supplemented by the Kodama flow [49], and the Kodama temperature (which reduces to $T_{\mathrm{H}}$ in the static case) [53]. Now, both of these prescriptions give back the Bekenstein-Hawking entropy in the static case $[49,50]$, which receives support from the more involved discussion of dynamical black hole horizons. Another indication of the privileged role of the Misner-Sharp-Hernandez mass among the spectrum of quasilocal masses available in the literature [40] comes from the fact that it is the Noether charge associated with the conservation of the Kodama current [48] (which always holds in spherical symmetry [53]). It is the Misner-SharpHernandez mass that is used as thermodynamical energy in the thermodynamics of dynamical apparent horizons: this is a self-consistent thermodynamical picture that uses in an essential way the Kodama time, in the absence of a timelike Killing vector (see Ref. [54] for a review of the relevant tunneling formalism). Replacing the black hole mass used in the thermodynamics of static black hole event horizons with another energy (quasilocal or not) would imply the same replacement in the thermodynamics of apparent horizons and would make it inconsistent.

Regarding the second question about the temperature, note that the Hawking radiation is obtained if the geometry with horizon is prescribed and the standard Hawking temperature is the parameter appearing in the thermal distribution of the emitted Hawking radiation. If we place the black hole in a heat bath at temperature $T$, thermal equilibrium between the black hole radiation and the heat bath occurs when the radiation temperature equals the temperature of the heat bath, $T=T_{\mathrm{H}}$. Therefore, we can use the heat bath as a thermometer. The temperature measured by the heat bath must be the standard Hawking temperature of the Hawking radiation and, therefore, we identify the latter with the black hole temperature.

Entropy constructs other than the Bekenstein-Hawking one are often considered in the literature, for example the Rényi entropy [25,31-33]

$$
\mathcal{S}_{\mathrm{R}}=\frac{1}{\alpha} \ln (1+\alpha \mathcal{S}),
$$

where $\alpha$ is a parameter and $\mathcal{S}$ is the Bekenstein-Hawking entropy (3.3), which is recovered in the limit $\alpha \rightarrow 0$. In this case, Eq. (3.2) with $\mathcal{S}_{0}=0$ gives 


$$
\mathcal{S}_{\mathrm{R}}=\frac{1}{\alpha} \ln \left(1+4 \pi \alpha G M^{2}\right) .
$$

If the mass $M$ coincides with the energy $E$ of the system due to the energy conservation as in [25,31-33], in order for this system to be consistent with the thermodynamical equation $d \mathcal{S}=d E / T$, one needs to define the "Rényi temperature" $T_{\mathrm{R}}$ by

$$
\frac{1}{T_{\mathrm{R}}} \equiv \frac{d \mathcal{S}_{\mathrm{R}}}{d M}=\frac{8 \pi G M}{1+4 \pi \alpha G M^{2}},
$$

that is,

$$
T_{\mathrm{R}}=\frac{1}{8 \pi G M}+\frac{\alpha M}{2}=T_{\mathrm{H}}+\frac{\alpha}{16 \pi G T_{\mathrm{H}}}
$$

which is, of course, quite different from the Hawking temperature $T_{\mathrm{H}}$. Therefore, this "Rényi temperature" $T_{\mathrm{R}}$ is not the temperature perceived by any observer detecting Hawking radiation, which is the concept that started the research area of black hole thermodynamics. This fact tells us that the "Rényi temperature" $T_{\mathrm{R}}$ is likely physically irrelevant for black hole thermodynamics.

In Eq. (3.13), we assumed that the thermodynamical energy $E$ is the black hole mass $M$ and we obtained an unphysical result. One wonders what the result would be if we assumed that the thermodynamical temperature $T$ coincides with the Hawking temperature $T_{\mathrm{H}}$, instead of assuming $E=M$.

Under the assumptions $T=T_{\mathrm{H}}$ and $\mathcal{S}=\mathcal{S}_{\mathrm{R}}$, using $d E=T d \mathcal{S}$ we find the corresponding thermodynamical energy $E_{\mathrm{R}}$ :

$$
\begin{aligned}
d E_{\mathrm{R}} & =T_{\mathrm{H}} d \mathcal{S}_{\mathrm{R}}=\frac{1}{8 \pi G M} \frac{8 \pi G M d M}{1+4 \pi \alpha G M^{2}} \\
& =\frac{d M}{1+4 \pi \alpha G M^{2}}
\end{aligned}
$$

and, integrating,

$E_{\mathrm{R}}=\frac{\arctan (\sqrt{4 \pi \alpha G} M)}{\sqrt{4 \pi \alpha G}}=M-\frac{4 \pi \alpha G M^{2}}{3}+\mathcal{O}\left(\alpha^{2}\right)$,

where we have fixed the integration constant so that $E_{\mathrm{R}}=0$ when $M=0$. Due to the correction $-\frac{4 \pi \alpha G M^{2}}{3}+\mathcal{O}\left(\alpha^{2}\right)$, the expression (3.17) of the thermodynamical energy $E_{\mathrm{R}}$ obtained differs from the black hole mass $M, E_{\mathrm{R}} \neq E$, in a way that has no obvious physical interpretation. More important, if the spherically symmetric dust shell collapses to a Schwarzschild black hole, the result obtained above seems to conflict with energy conservation.
Another entropy notion encountered frequently in the literature and motivated by nonextensive statistics is the Tsallis entropy [39], which suggests as a potential alternative to the Bekenstein-Hawking entropy for black holes the quantity

$$
\mathcal{S}_{\mathrm{T}}=\frac{A_{0}}{4 G}\left(\frac{A}{A_{0}}\right)^{\delta}
$$

instead of the Rényi-like entropy (3.11) ([34], see also [55]). Here $A_{0}$ is a constant with the dimensions of a length squared and $\delta$ is a parameter that quantifies the nonextensivity. In the case $\delta=1$, we obtain the standard Bekenstein-Hawking entropy (2.26) or (3.3).

In Ref. [55], cosmology with the Tsallis-type entropy (3.18) has been studied. The corresponding late-time universe contains an effective dark energy, which could be phantom or quintessence, without an effective cosmological constant. One obtains an effective cosmological constant from the generalized nonextensive Tsallis type entropy also in the inflationary era of the early universe.

As done in Eq. (3.13), assuming that the thermodynamical energy $E$ is given by the black hole mass $M$, we find $A=4 \pi(2 G M)^{2}=16 \pi G^{2} E^{2}$ and the corresponding Tsallis entropy

$$
\mathcal{S}_{\mathrm{T}}=\frac{A_{0}}{4 G}\left(\frac{16 \pi G^{2} E^{2}}{A_{0}}\right)^{\delta}
$$

We may define also the "Tsallis temperature"

$$
\begin{aligned}
T_{\mathrm{T}} & \equiv \frac{d E}{d \mathcal{S}_{\mathrm{T}}}=\frac{2 G}{\delta A_{0} E^{2 \delta-1}}\left(\frac{A_{0}}{16 \pi G^{2}}\right)^{\delta} \\
& =\frac{2 G}{\delta A_{0} M^{2 \delta-1}}\left(\frac{A_{0}}{16 \pi G^{2}}\right)^{\delta}
\end{aligned}
$$

which is, of course, different from the Hawking temperature (2.9) unless $\delta=1$. Then, instead of identifying the black hole mass $M$ with the thermodynamical energy $E$, we could assume that the temperature is the Hawking temperature (2.9). Then, since $A=4 \pi\left(4 \pi T_{\mathrm{H}}\right)^{-2}=\frac{1}{4 \pi T_{\mathrm{H}}^{2}}$, we would find

$$
\mathcal{S}_{\mathrm{T}}=\frac{A_{0}^{1-\delta}}{4 G\left(4 \pi T_{\mathrm{H}}^{2}\right)^{\delta}}
$$

and then we may define the "Tsallis energy" $E_{\mathrm{T}}$ by

$$
d E_{\mathrm{T}}=T_{\mathrm{H}} d \mathcal{S}_{\mathrm{T}}=-\frac{\delta A_{0}{ }^{1-\delta} d T_{\mathrm{H}}}{2 G(4 \pi)^{\delta} T_{\mathrm{H}}{ }^{2 \delta}}
$$

or, integrating, 


$$
E_{\mathrm{T}}=\frac{\delta A_{0}{ }^{1-\delta}}{2(2 \delta-1) G(4 \pi)^{\delta} T_{\mathrm{H}}^{2 \delta-1}}=\frac{\delta A_{0}^{1-\delta}(8 \pi G M)^{2 \delta-1}}{2(2 \delta-1) G(4 \pi)^{\delta}}
$$

where we have again fixed the integration constant by imposing $E_{\mathrm{T}}=0$ at $M=0$. The standard relation $E_{\mathrm{T}}=M$ is reproduced for $\delta=1$, but $E_{\mathrm{T}} \neq M$ otherwise.

In order to build a thermodynamic theory one needs to identify, in addition to the entropy, the thermodynamical energy $E$ and the temperature $T$. If we choose as entropy any quantity different from the Bekenstein-Hawking entropy, then we lack physical grounds and we can choose the thermodynamical energy $E$ and/or the temperature $T$ as we wish. Not surprisingly, this procedure leads to an unphysical outcome, i.e., it conflicts with energy conservation and/or with the established physics of the Hawking radiation process. To conclude, the assumed Rényi entropy (3.11) cannot be the black hole entropy, although it might be the entropy of a system different from the black hole (see, for example, Ref. [26]), as in the case of the Tsallis entropy. The Tsallis entropy is obtained as a result of the Fermi, Bose, or Boltzmann statistics for the system with long-range forces by the standard statistical mechanics procedure of using the Hamiltonian and counting the number of states. In this sense, there is no physical counterpart that could be the Rényi statistics. The Rényi entropy could be an index specifying the information, with no relation with the statistics of any physical system.

\section{DISCUSSION AND CONCLUSIONS}

The idea of replacing the standard Boltzmann-Gibbs statistics with nonextensive statistics has led to the Rényi [38] and Tsallis [39] entropies. Since the BekensteinHawking black hole entropy is nonextensive, several authors have considered the possibility of replacing the Bekenstein-Hawking entropy with the Rényi or the Tsallis one. This proposal has somehow mixed with the parallel idea of correcting the Bekenstein-Hawking entropy with modifications due to the Generalized Uncertainty Principle, or with other ideas. We have pointed out the fact that changing the entropy goes hand in hand with changing other thermodynamical quantities, or else the entire thermodynamical theory may become inconsistent, but this creates more problems of principle.

For spherical black holes, one could think of reconciling the Hawking temperature $T_{\mathrm{H}}$ with some entropy notion different from the Bekenstein-Hawking entropy by adopting as thermodynamical energy some quasilocal energy $E_{x}$, for which various prescriptions can be found in the literature [40]. We have discussed the Brown-York energy as an example. However, already at first sight, this task appears very difficult to say the least (and, even if it was logically possible, very contrived from the physical point of view). The explicit forms of the Rényi and Tsallis entropies make it practically impossible to match $T_{\mathrm{H}}, E_{x}$, and $\mathcal{S}_{\mathrm{R}}$ (or $\mathcal{S}_{\mathrm{T}}$ ) while satisfying the relation $T d \mathcal{S}_{\mathrm{R}, \mathrm{T}}=d E_{x}$ even for the simple Schwarzschild black hole, which is static and isolated. Indeed, the case for the Bekenstein-Hawking entropy seems rather compelling at this point.

Let us examine now possible loopholes to the area law for entropy. In the previous sections, we have shown that the area law of the Bekenstein-Hawking entropy can always be obtained if we assume that the thermodynamical energy $E$ is identified with the mass $M, E=M$, and the temperature of the system is the Hawking temperature. Moreover, other forms of the entropy such as the Rényi and the Tsallis entropies lead to unphysical results.

In order to show that $E=M$, we have used the argument of the dust shell collapse in conjunction with energy conservation. This argument might not carry over to theories of modified gravity, especially higher derivative theories such as $F(R)$ gravity. In the Einstein gravity, the falling shell of the dust constitutes an exact solution of the field equations. In higher derivative gravity, the junction conditions between the matter and the vacuum at the shell becomes more complex and more restrictive [56-65]. Therefore, whether the infalling dust shell constitutes an exact solution of the field equations remains to be determined and the previous argument may not apply. What, is more the gravitational constant $G$ becomes a scalar degree of freedom $\phi \sim G^{-1}$, the Brans-Dicke-like scalar field [66], already in scalar-tensor gravity (which includes $F(R)$ theories as a subclass [67-69]). Then, the area law for the entropy becomes $\mathcal{S}=\phi A / 4$ and the scalar $\phi$ gives a contribution to the differential $d \mathcal{S}$ in $T d \mathcal{S}=d E$. While it is true that all vacuum black holes of scalar-tensor gravity that are spherical, asymptotically flat, static, and sit in a minimum of the potential $V(\phi)$ for $\phi$ reduce to the Schwarzschild black hole [70-73], the discussion becomes more complicated already for asymptotically de Sitter black holes.

Even in Einstein gravity, if we include quantum corrections there might be modifications to the reasoning above. For example, quantum fluctuations of the horizon have been discussed in [74]. The quantum fluctuation may effectively increase the area of the horizon, changing the entropy. In the arguments following Eq. (2.11) we have used the WKB approximation, which is valid at low temperature. But at high temperature the correction given by the $1 / T_{\mathrm{H}}$ expansion becomes large and there could be a violation of the entropy area law. The $1 / T_{\mathrm{H}}$ correction may be also regarded as a quantum corrections because if we include $\hbar$, the factor $t_{0}=1 / T_{\mathrm{H}}$ in front of the Hamiltonian $H$ in (2.8) becomes $\frac{t_{0}}{\hbar}=\frac{1}{\hbar T_{\mathrm{H}}}$. Such corrections are considered in [75]. Then, if we assume $E=M$ as in (3.13), the Hawking temperature might not be modified and the thermal distribution of the radiation might be changed from that of blackbody radiation. Such a correction might appear as the shift from $\alpha \rightarrow 0$ in the Rényi-type 
entropy (3.11) or the shift from $\delta=1$ in the Tsallis-like entropy (3.18).

In general, entropy corresponds to the number of physical degrees of freedom of a thermodynamical system. The renormalization of a quantum theory implies that the number of degrees of freedom depends on the scale. In standard field theory massive modes decouple in the lowenergy regime and, therefore, the number of degrees of freedom decreases. The situation is more complicated in the case of gravity: if gravity is described by string theory, an infinite tower of massive modes appears at high temperature, which introduces the upper bound to the temperature called Hagedorn temperature. Even from a naive point of view, if the spacetime fluctuations become large in the high temperature regime, the number of degrees of freedom may increase. If, instead, gravity becomes topological, the number of degrees of freedom will decrease, which could be consistent with holography. In the case of the Bekenstein-Hawking entropy (3.3) or (2.26), because the area is given by $A=\frac{1}{4 \pi T_{\mathrm{H}}}$, in the high temperature regime of large $T_{\mathrm{H}}$, the entropy decreases. This happens, of course, because the smaller black hole has higher Hawking temperature. The decrease in the entropy corresponds to the loss of physical degrees of freedom due to Hawking radiation.

In the case of the Renyi-type entropy (3.11), if the parameter $\alpha$ is positive, the deviation from the BekensteinHawking entropy $\mathcal{S}$ (3.3) becomes large in the lowtemperature region where $\mathcal{S}$ becomes large, which might contradict the above speculations in which this deviation becomes large in the high-temperature region instead. If $\alpha<0$, we may further modify the Rényi-like entropy as

$$
\mathcal{S}_{\mathrm{R}}=\frac{1}{\alpha} \ln |1+\alpha \mathcal{S}|
$$

then there is a singularity when $1+\alpha \mathcal{S}=0$, or

$$
T_{\mathrm{H}}=T_{\mathrm{c}} \equiv \sqrt{-\frac{\alpha}{16 \pi G}} .
$$

If we start at temperature higher than $T_{\mathrm{c}}$, the critical temperature $T_{\mathrm{c}}$ gives a lower bound on the Hawking temperature. If, instead, we start at temperature lower than $T_{\mathrm{c}}$, then $T_{\mathrm{c}}$ sets an upper bound that might correspond to the Hagedorn temperature. Therefore, it might be interesting to consider a model in which $\alpha$ depends on the temperature and becomes negative at high temperature. In the case of the Tsallis-like entropy (3.18), if the parameter $\delta$ is larger than unity, $\delta>1$, the Tsallis entropy (3.18) becomes smaller than the Bekenstein-Hawking entropy at high temperature. If $\delta<1$, the Tsallis type entropy becomes larger than the Bekenstein-Hawking entropy at high temperatures. Then, it might be interesting to consider a model in which the parameter $\delta$ depends on the temperature. If $\delta>1$ at high temperatures, this model might correspond to the model becoming topological at high $T$. If, instead, $\delta<1$ in the high-temperature regime, the model might correspond to a violent fluctuation of spacetime or to string theory.

If the Rényi entropy (3.11) comes from a quantum correction, we may express its parameter as $\alpha=\alpha_{0} \hbar$ and expand Eq. (3.11) with respect to $\hbar$ as

$\mathcal{S}_{\mathrm{R}}=\frac{1}{\alpha_{0} \hbar} \ln \left(1+\alpha_{0} \hbar \mathcal{S}\right)=\mathcal{S}-\frac{\alpha_{0} \hbar}{2} \mathcal{S}^{2}+\frac{\alpha_{0}^{2} \hbar^{2}}{3} \mathcal{S}^{3}+\cdots$

If $\alpha$ is positive, the leading correction is negative, which could hint at gravity becoming topological by quantum effects, whereas if $\alpha<0$ the leading correction is positive, possibly saying that the number of degrees of freedom increases as in string theory. Similarly, by writing $\delta=1+\delta_{0} \hbar$, we may expand the Tsallis entropy (3.18) as

$$
\begin{aligned}
\mathcal{S}_{\mathrm{T}} & =\frac{A_{0}}{4 G}\left(\frac{A}{A_{0}}\right)^{1+\delta_{0} \hbar} \\
& =\frac{A}{4 G}\left[1+\delta_{0} \hbar \ln \frac{A}{A_{0}}+\frac{\delta_{0}^{2} \hbar^{2}}{2 !}\left(\ln \frac{A}{A_{0}}\right)^{2}+\cdots\right] .
\end{aligned}
$$

Then, if $\delta>0$ the number of degrees of freedom increases for large black holes where $A>A_{0}$ and decreases for small black holes with $A<A_{0}$. If $\delta<0$, the number of degrees of freedom increases for small black holes and decreases for large ones. We should also note that the logarithmic correction in Eq. (4.4) often appears at the first loop in quantum field theory.

Another possible loophole might be the case in which there are two horizons, as discussed in Ref. [76], where the entropies of the Reissner-Nordström and of the Kerr black holes have been investigated, and it is claimed that there might be contributions to the entropy from the correlation of the two horizons. As another example, we may consider the Schwarzschild-de Sitter/Kottler spacetime, where two horizons (the black hole and the cosmological horizon) appear. The line element is

$$
\begin{gathered}
d s^{2}=-f(r) d t^{2}+\frac{d r^{2}}{f(r)}+r^{2} d \Omega_{(2)}^{2}, \\
f(r)=1-\frac{2 G M}{r}-\frac{r^{2}}{l^{2}}
\end{gathered}
$$

if we assume $l^{2}>0$, we can rewrite $f(r)$ as

$$
\begin{gathered}
f(r)=-\frac{\left(r-r_{-}\right)\left(r-r_{+}\right)\left(r+r_{+}+r_{-}\right)}{l^{2} r}, \\
r_{+} r_{-}\left(r_{+}+r_{-}\right)=2 G M l^{2}, \\
r_{+}^{2}+r_{-}^{2}+r_{+} r_{-}=l^{2},
\end{gathered}
$$


where we assume $r_{+}>r_{-}$. Then, $r_{+}$and $r_{-}$are the radii of the cosmological and black hole horizons, respectively. When $r \sim r_{-}, f(r)$ behaves as

$$
f(r) \sim \frac{\left(r_{+}-r_{-}\right)\left(2 r_{-}+r_{+}\right)}{l^{2} r_{-}}\left(r-r_{-}\right),
$$

and the Hawking temperature $T_{\mathrm{H}}^{(\mathrm{bh})}$ of the black hole horizon is

$$
\begin{aligned}
T_{\mathrm{H}}^{(\mathrm{bh})} & =\frac{\left(r_{+}-r_{-}\right)\left(2 r_{-}+r_{+}\right)}{4 \pi l^{2} r_{-}} \\
& =\frac{1}{4 \pi l^{2}} \frac{-2 r_{-}^{2}+r_{-} r_{+}+r_{+}^{2}}{r_{-}}=\frac{1}{4 \pi l^{2}} \frac{l^{2}-3 r_{-}^{2}}{r_{-}} \\
& =\frac{1}{4 \pi l^{2}}\left(\frac{l^{2}}{r_{-}}-3 r_{-}\right) .
\end{aligned}
$$

Similarly, the Gibbons-Hawking temperature of the cosmological horizon is [77]

$$
T_{\mathrm{H}}^{(\mathrm{c})}=\frac{\left(r_{+}-r_{-}\right)\left(r_{-}+2 r_{+}\right)}{4 \pi l^{2} r_{+}}
$$

and we should note that

$$
T_{\mathrm{H}}^{(\mathrm{bh})}-T_{\mathrm{H}}^{(\mathrm{c})}=\frac{\left(r_{+}-r_{-}\right)^{2}\left(r_{+}+r_{-}\right)}{4 \pi l^{2} r_{+} r_{-}} \geq 0
$$

which implies that there could be heat flow from the black hole to the cosmological horizon.

In Eq. (4.13), the equality "=" holds if and only if $r_{+}=r_{-}$, that is, in the extremal case corresponding to the Nariai spacetime. Because $T_{\mathrm{H}}^{(\mathrm{bh})} \neq T_{\mathrm{H}}^{(\mathrm{c})}$ in general, even if we Wick-rotate the spacetime into the Euclidean signature, we cannot remove both conical singularities corresponding to the black hole and the cosmological horizons and the calculation of the free energy performed for the Schwarzschild-anti-de Sitter spacetime could not be applied. In the following, we consider the case in which only the conical singularity of the black hole horizon is removed by assuming the period of the Euclidean time $1 / T_{\mathrm{H}}^{(\mathrm{bh})}$, but the conical singularity of the cosmological horizon remains.

The equations (4.8), (4.9) tell us that

$$
\begin{array}{r}
r_{-}\left(2 r_{+}+r_{-}\right) \frac{d r_{+}}{d M}+r_{+}\left(r_{+}+2 r_{-}\right) \frac{d r_{-}}{d M}=2 G l^{2}, \\
\left(2 r_{+}+r_{-}\right) \frac{d r_{+}}{d M}+\left(r_{+}+2 r_{-}\right) \frac{d r_{-}}{d M}=0,
\end{array}
$$

which give

$$
\begin{gathered}
\frac{d r_{+}}{d M}=-\frac{2 G l^{2}}{\left(r_{-}-r_{+}\right)\left(2 r_{+}+r_{-}\right)} \\
\frac{d r_{-}}{d M}=-\frac{2 G l^{2}}{\left(r_{+}-r_{-}\right)\left(r_{+}+2 r_{-}\right)}=-\frac{2 G l^{2}}{r_{+}^{2}+r_{+} r_{-}-2 r_{-}^{2}} \\
=-\frac{2 G l^{2}}{l^{2}-3 r_{-}^{2}} .
\end{gathered}
$$

Then the action is

$$
\begin{aligned}
S & =\frac{1}{16 \pi G} \int d^{4} x \sqrt{-g}\left(R-\frac{6}{l^{2}}\right) \\
& =\frac{3}{2 G l^{2}} \int_{0}^{1 / T_{\mathrm{H}}^{(\mathrm{bh})}} d t \int_{r_{-}}^{r^{+}} d r r^{2}=\frac{r_{+}^{3}-r_{-}^{3}}{2 G l^{2} T_{\mathrm{H}}} \\
& =\frac{\left(r_{+}-r_{-}\right)\left(r_{+}^{2}+r_{-}^{2}+r_{+} r_{-}\right)}{2 G l^{2} T_{\mathrm{H}}^{\mathrm{bh}}}=\frac{r_{+}-r_{-}}{2 G T_{\mathrm{H}}^{\mathrm{bh}}}
\end{aligned}
$$

and the free energy $F$ reads

$$
F=T_{\mathrm{H}}^{(\mathrm{bh})} S=\frac{r_{+}-r_{-}}{2 G} .
$$

By using the thermodynamical relations for the thermodynamical energy $E$ and the entropy $\mathcal{S}$ in (2.24), we then find

$$
\begin{aligned}
\mathcal{S} & =-\frac{1}{2 G} \frac{-\frac{2 G l^{2}}{\left(r_{-}-r_{+}\right)\left(2 r_{+}+r_{-}\right)}+\frac{2 G l^{2}}{\left(r_{+}-r_{-}\right)\left(r_{+}+2 r_{-}\right)}}{-\frac{1}{4 \pi l^{2}}\left(-\frac{l^{2}}{r_{-}^{2}}-3\right) \frac{2 G l^{2}}{l^{2}-3 r_{-}^{2}}} \\
& =-\frac{2 \pi l^{2} r_{-}^{2}}{G} \frac{2 r_{-}+r_{+}+2 r_{+}+r_{-}}{\left(r_{+}-r_{-}\right)\left(r_{+}+2 r_{-}\right)\left(2 r_{+}+r_{-}\right)} \frac{l^{2}-3 r_{-}^{2}}{l^{2}+3 r_{-}^{2}} \\
& =-\frac{6 \pi l^{2} r_{-}^{2}}{G} \frac{r_{+}+r_{-}}{\left(2 r_{+}+r_{-}\right)\left(l^{2}+3 r_{-}^{2}\right)},
\end{aligned}
$$

which is negative and, as a result, the quantity $\mathcal{S}$ given by Eq. (4.21) cannot be identified with the entropy. We cannot obtain the area law entropy for the Schwarzschild-de Sitter spacetime, which might be due to the correlation between the two horizons.

A possible way to avoid the above problem of the Schwarzschild-de Sitter spacetime could be to analytically continue the results (2.24) and (2.26) to the Schwarzschildanti-de Sitter spacetime by replacing $l^{2} \rightarrow-l^{2}$, obtaining

$$
F=-\frac{r_{\mathrm{H}}\left(r_{-}{ }^{2}+l^{2}\right)}{4 G l^{2}}, \quad \mathcal{S}=\frac{-\frac{3 r_{-}{ }^{2}+l^{2}}{4 G l^{2}}}{-\frac{1}{4 \pi l^{2}}\left(3+\frac{l^{2}}{r_{-}{ }^{2}}\right)}=\frac{A}{4 G},
$$

which could be the standard result. The differences between Eqs. (4.21) and (4.22) originate from the integration region of the action, as we find by comparing Eqs. (2.19) 
and (4.18) and also come from the subtraction of the background in (2.23). This fact tells us that, if we can use the analytic continuation $l^{2} \rightarrow-l^{2}$, the domain of integration should include the region outside the cosmological horizon $L \gg r_{+}$, which could induce a conceptual problem in black hole physics. Anyway, the existence of two horizons makes it difficult to judge what could be the correct prescription to evaluate the entropy.

In [55], the holographic dark energy model based on the Tsallis-type entropy or its generalization associated with the cosmological horizon was proposed. We may consider the creation of a black hole in the holographic dark energy. If the holographic dark energy has a higher temperature than the black hole temperature, there could occur an energy flow to the black hole and the black hole may consequently grow, which may signal an instability of the holographic dark energy. Black hole seeds may be generated by fluctuations of the energy density, which could be the density of the holographic dark energy. If we regard the holographic dark energy as a kind of effective perfect fluid, however, it should have a negative effective pressure. This negative pressure generates a repulsion in the fluid, which protects a fluctuation from growing and becoming a black hole. In this sense, the collapse of the holographic dark energy to black holes could be avoided and the holographic dark energy could be stable.
The flow of heat energy onto a black hole, if it occurs due to the temperature difference between black hole and surrounding dark energy, is difficult to model. Ref. [78] models the spherical accretion of phantom dark energy onto a black hole in the test fluid approximation but, realistically, the backreaction is not described and the same will be true for radial heat flow. It may be possible to describe the net heat transferred in an event occurring between two stationary phases, but this would probably require a cosmological evolution that is completely ad hoc.

To conclude, the entropy area law is physically well motivated, unlike its Rényi and Tsallis potential competitors, and solid motivations seem to be needed before departures from it can be taken too seriously. In particular, replacing the Bekenstein-Hawking entropy with the Rényi, Tsallis, or another entropy is premature and rather arbitrary at the current stage of knowledge.

\section{ACKNOWLEDGMENTS}

This work was also partially supported by the Kazan Federal University Strategic Academic Leadership Program (S. D. O), by JSPS Grant-in-Aid for Scientific Research (C) No. 18K03615 (S. N.), and by the Natural Sciences \& Engineering Research Council of Canada, Grant No. 2016-03803 (V. F.).
[1] J. D. Bekenstein, Phys. Rev. D 7, 2333 (1973).

[2] S. W. Hawking, Commun. Math. Phys. 43, 199 (1975); 46, 206(E) (1976).

[3] J. M. Bardeen, B. Carter, and S. W. Hawking, Commun. Math. Phys. 31, 161 (1973).

[4] R. M. Wald, Living Rev. Relativity 4, 6 (2001).

[5] R. M. Wald, General Relativity (Chicago University Press, Chicago, 1984).

[6] S. Carlip, Int. J. Mod. Phys. D 23, 1430023 (2014).

[7] S. W. Hawking and D. N. Page, Commun. Math. Phys. 87, 577 (1983).

[8] J. M. Maldacena, Adv. Theor. Math. Phys. 2, 231 (1998).

[9] E. Witten, Adv. Theor. Math. Phys. 2, 505 (1998).

[10] D. Kastor, S. Ray, and J. Traschen, Classical Quantum Gravity 26, 195011 (2009).

[11] D. Kubiznak and R. B. Mann, Can. J. Phys. 93, 999 (2015).

[12] A. Karch and B. Robinson, J. High Energy Phys. 12 (2015) 073.

[13] D. Kubiznak and F. Simovic, Classical Quantum Gravity 33, 245001 (2016).

[14] D. Kubiznak, R. B. Mann, and M. Teo, Classical Quantum Gravity 34, 063001 (2017).

[15] M. Sinamuli and R. B. Mann, Phys. Rev. D 96, 086008 (2017).

[16] A. G. Tzikas, Phys. Lett. B 788, 219 (2019).
[17] D. Astefanesei, R. B. Mann, and R. Rojas, J. High Energy Phys. 11 (2019) 043.

[18] M. Mir, R. A. Hennigar, J. Ahmed, and R. B. Mann, J. High Energy Phys. 08 (2019) 068.

[19] A. Kumar, S. G. Ghosh, and S. D. Maharaj, Phys. Dark Universe 30, 100634 (2020).

[20] C. Tsallis and L. J. L. Cirto, Eur. Phys. J. C 73, 2487 (2013).

[21] A. Bialas and W. Czyz, Europhys. Lett. 83, 60009 (2008).

[22] X. Huang and Y. Zhou, J. High Energy Phys. 02 (2015) 068.

[23] R. Brustein and A. J. M. Medved, Phys. Rev. D 91, 084062 (2015).

[24] T. Nishioka, J. High Energy Phys. 07 (2014) 061.

[25] V. G. Czinner and H. Iguchi, Phys. Lett. B 752, 306 (2016).

[26] X. Dong, Nat. Commun. 7, 12472 (2016).

[27] W. Y. Wen, Int. J. Mod. Phys. D 26, 1750106 (2017).

[28] V. G. Czinner and H. Iguchi, Eur. Phys. J. C 77, 892 (2017).

[29] C. V. Johnson, Int. J. Mod. Phys. D 28, 1950091 (2019).

[30] S. Qolibikloo and A. Ghodsi, Eur. Phys. J. C 79, 406 (2019).

[31] L. Tannukij, P. Wongjun, E. Hirunsirisawat, T. Deesuwan, and C. Promsiri, Eur. Phys. J. Plus 135, 500 (2020).

[32] C. Promsiri, E. Hirunsirisawat, and W. Liewrian, Phys. Rev. D 102, 064014 (2020).

[33] D. Samart and P. Channuie, arXiv:2012.14828 [Phys. Rev. D (to be published)].

[34] J. Ren, J. High Energy Phys. 05 (2021) 080. 
[35] K. Mejrhit and R. Hajji, Eur. Phys. J. C 80, 1060 (2020).

[36] R. Nakarachinda, E. Hirunsirisawat, L. Tannukij, and P. Wongjun, Phys. Rev. D 104, 064003 (2021).

[37] E. M. C. Abreu and J. Ananias Neto, Europhys. Lett. 133, 49001 (2021).

[38] A. Rényi, On measures of information and entropy, in Proceedings of the 4th Berkeley Symposium on Mathematics, Statistics and Probability, 1960, Vol. I (University of California Press, Berkeley and Los Angeles, 1960), pp. 547-561.

[39] C. Tsallis, J. Stat. Phys. 52, 479 (1988).

[40] L. B. Szabados, Living Rev. Relativity 12, 4 (2009).

[41] C. W. Misner and D. H. Sharp, Phys. Rev. 136, B571 (1964).

[42] W. C. Hernandez and C. W. Misner, Astrophys. J. 143, 452 (1966).

[43] J. D. Brown and J. W. York, Jr., Phys. Rev. D 47, 1407 (1993).

[44] G. C. McVittie, Mon. Not. R. Astron. Soc. 93, 325 (1933).

[45] M. Carrera and D. Giulini, Phys. Rev. D 81, 043521 (2010).

[46] V. Faraoni, Cosmological and Black Hole Apparent Horizons, Lect. Notes Phys. Vol. 907 (Springer, New York, 2015).

[47] J. Sultana and C. C. Dyer, Gen. Relativ. Gravit. 37, 1347 (2005).

[48] S. A. Hayward, Phys. Rev. D 53, 1938 (1996).

[49] S. A. Hayward, S. Mukohyama, and M. C. Ashworth, Phys. Lett. A 256, 347 (1999).

[50] S. A. Hayward, Classical Quantum Gravity 15, 3147 (1998).

[51] R. M. Wald, Phys. Rev. D 48, R3427 (1993).

[52] V. Iyer and R. M. Wald, Phys. Rev. D 50, 846 (1994).

[53] H. Kodama, Prog. Theor. Phys. 63, 1217 (1980).

[54] L. Vanzo, G. Acquaviva, and R. Di Criscienzo, Classical Quantum Gravity 28, 183001 (2011).

[55] S. Nojiri, S. D. Odintsov, and E. N. Saridakis, Eur. Phys. J. C 79, 242 (2019).

[56] C. Barrabes and G.F. Bressange, Classical Quantum Gravity 14, 805 (1997).
[57] G. F. Bressange, Classical Quantum Gravity 17, 2509 (2000).

[58] J. Velay-Vitow and A. DeBenedictis, Phys. Rev. D 96, 024055 (2017).

[59] V. Berezin, V. Dokuchaev, Y. Eroshenko, and A. Smirnov, Int. J. Mod. Phys. A 35, 2040002 (2020).

[60] V. A. Berezin, V. I. Dokuchaev, Y. N. Eroshenko, and A. L. Smirnov, Classical Quantum Gravity 38, 045014 (2021).

[61] V. A. Berezin, V. I. Dokuchaev, Y. N. Eroshenko, and A. L. Smirnov, Phys. Part. Nucl. 51, 730 (2020).

[62] L. Avilés, H. Maeda, and C. Martinez, Classical Quantum Gravity 37, 075022 (2020).

[63] G. J. Olmo and D. Rubiera-Garcia, Classical Quantum Gravity 37, 215002 (2020).

[64] C. S. Chu and H. S. Tan, arXiv:2103.06314 [Phys. Rev. D (to be published)].

[65] J. L. Rosa, Phys. Rev. D 103, 104069 (2021).

[66] C. Brans and R. H. Dicke, Phys. Rev. 124, 925 (1961).

[67] T. P. Sotiriou and V. Faraoni, Rev. Mod. Phys. 82, 451 (2010).

[68] S. Nojiri, S. D. Odintsov, and V. K. Oikonomou, Phys. Rep. 692, 1 (2017).

[69] S. Nojiri and S. D. Odintsov, Phys. Rep. 505, 59 (2011).

[70] S. W. Hawking, Commun. Math. Phys. 25, 167 (1972).

[71] J. D. Bekenstein, arXiv:gr-qc/9605059.

[72] T. P. Sotiriou and V. Faraoni, Phys. Rev. Lett. 108, 081103 (2012).

[73] S. Bhattacharya, K. F. Dialektopoulos, A. E. Romano, and T. N. Tomaras, Phys. Rev. Lett. 115, 181104 (2015).

[74] J. D. Barrow, Phys. Lett. B 808, 135643 (2020).

[75] G. Cognola, E. Elizalde, S. Nojiri, S. D. Odintsov, and S. Zerbini, J. Cosmol. Astropart. Phys. 02 (2005) 010.

[76] G. E. Volovik, arXiv:2107.11193.

[77] G. W. Gibbons and S. W. Hawking, Phys. Rev. D 15, 2738 (1977).

[78] E. Babichev, V. Dokuchaev, and Y. Eroshenko, Phys. Rev. Lett. 93, 021102 (2004). 